\title{
THE PRADER-WILLI SYNDROME AND INTERSTITIAL DELETION OF CHROMOSOME 15: HIGH-RESOLUTION CHROMOSOME ANALYSES OF 14 PATIENTS WITH THE PRADER-WILLI SYNDROME AND OF 5 SUSPECTED INFANTS
}

\author{
Yoshimitsu FukUSHImA, ${ }^{1}$ Norio NirkawA, ${ }^{2}$ and Yoshikazu KurokI ${ }^{1}$ \\ ${ }^{1}$ Division of Medical Genetics, Kanagawa Children's Medical Center, \\ 2-138-4, Mutsukawa, Minami-ku, Yokohama 232, Japan \\ 2Department of Pediatrics, Hokkaido University School of Medicine, \\ $N-15, W-7$, Kita-ku, Sapporo 060, Japan
}

\begin{abstract}
Summary Fourteen patients with the Prader-Willi syndrome and five suspected infants were studied cytogenetically. High-resolution chromosome analyses with a modified ethidium bromide technique revealed an interstitial deletion of the proximal $15 \mathrm{q}$ in 13 out of the 14 typical patients and in four of the five suspected infants. A 15;15 Robertsonian translocation with a deletion in the proximal $15 \mathrm{q}$ was noticed in one infant. But in one patient, a normal karyotype was confirmed. The affected region common to all the patients was confined to a sub-band $15 \mathrm{q} 11.2$ (ISCN, 1981). These findings support the result of a recent study by Ledbetter et al. $(1981,1982)$, and indicate that the frequency of the $15 q$ deletion in definite patients with the Prader-Willi syndrome is very high.
\end{abstract}

\section{INTRODUCTION}

The Prader-Willi syndrome (PWS) is a disorder characterized by hypotonia and feeding difficulty in infancy followed by polyphagia, obesity, short stature, hypogonadism and mental retardation throughout the remainder of life. Although an autosomal-recessive mode of inheritance is suggested (McKusick, 1978), the etiology of the syndrome remains open. Previous cytogenetic studies in several cases showed various chromosome imbalances in which a chromosome 15 was frequently involved (Kouseff, 1982). With a recently developed high-resolution chromosome banding technique, Ledbetter et al. $(1981,1982)$ successfully demonstrated an interstitial deletion of a chromosome 15 affecting the q11-12 region as a cause of the syndrome in 23 of their 45 patients with PWS.

In order to investigate the relationship between PWS and the $15 \mathrm{q}$ deletion, we 
performed high-resolution chromosome analyses in 14 Japanese patients with typical PWS.

\section{MATERIALS AND METHODS}

Peripheral blood for chromosome analysis was obtained from 14 patients with PWS, five suspected infants and eleven of their parents. All 14 patients (Case 1 through 14), ten males and four females, aged 2 to 12 years, had essential symptoms of PWS (Holm, 1981) that included infantile hypotonia, hypogonadism, obesity, mental retardation, a peculiar facies characterized by almond-shaped eyes and triangular mouth, and short stature considering genetic background (Table 1). The presence of hypogonadism had not yet been confirmed in female patients. Five infants (Case 15 through 19) had been suspected to have PWS because they had muscular hypotonia, dysmorphic facial features including up-slanting almond eyes and a carp mouth, small hands and feet, and undescended testes in male infants. But the diagnosis was not conclusive clinically. Parents of Cases 3, 8, 15, 18, and 19 and the father of Case 7 were also examined cytogenetically.

Chromosome preparations were made by three-day culture method of peripheral lymphocytes. Prometaphase plates in more than 550-band stage (ISCN, 1981) were collected with a modified ethidium bromide technique (Ikeuchi and Sasaki, 1979). In short, peripheral lymphocytes were treated with $10 \mu \mathrm{g} / \mathrm{ml}$ of ethidium bromide (SIGMA) for two hours and with $0.02 \mu \mathrm{g} / \mathrm{ml}$ of Colcemid (GIBCO) for one hour prior to harvesting. Chromosomes were GTG-banded and then photographed.

Particular attention was paid to a pair of chromosome 15. Because of the small size of a deletion, the decision of a deletion of q11.2 or q12 bands was made on the basis of a consistent absence of these bands in five or more cells.

\section{RESULTS}

Thirteen of the 14 patients and four of the five infants had a deletion in the proximal 15q. Five of these (Cases 4, 7, 8, 9 and 13) lost q11.2 and q12 bands (Fig. 1), and twelve (Cases 1, 2, 3, 5, 6, 11, 12, 14, 15, 16, 17 and 18) lost only a q11.2 band (Fig. 2). One male infant (Case 19) had a 15;15 Robertsonian translocation with a

Fig. 1. High-resolution G-banded chromosome 15 from Case 9. The left homolog is normal but the right one is affected with a deletion in $15 q 11.2$ and 12 bands. Arrows on the chromosome and on the ideogram show breaking points.

Fig. 2. Chromosome 15 from Case 3. Deleted region is confined within a $15 q 11.2$ band.

Fig. 3. Chromosome 15 from Case 19 showing a 15;15 Robertsonian translocation with a deletion in $15 \mathrm{q} 11.2$ and $\mathrm{q} 12$ bands. Small arrows show break-points. 
Fig. 1

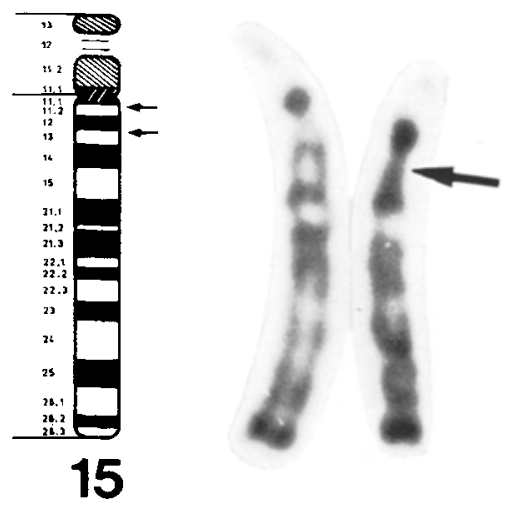

Fig. 2
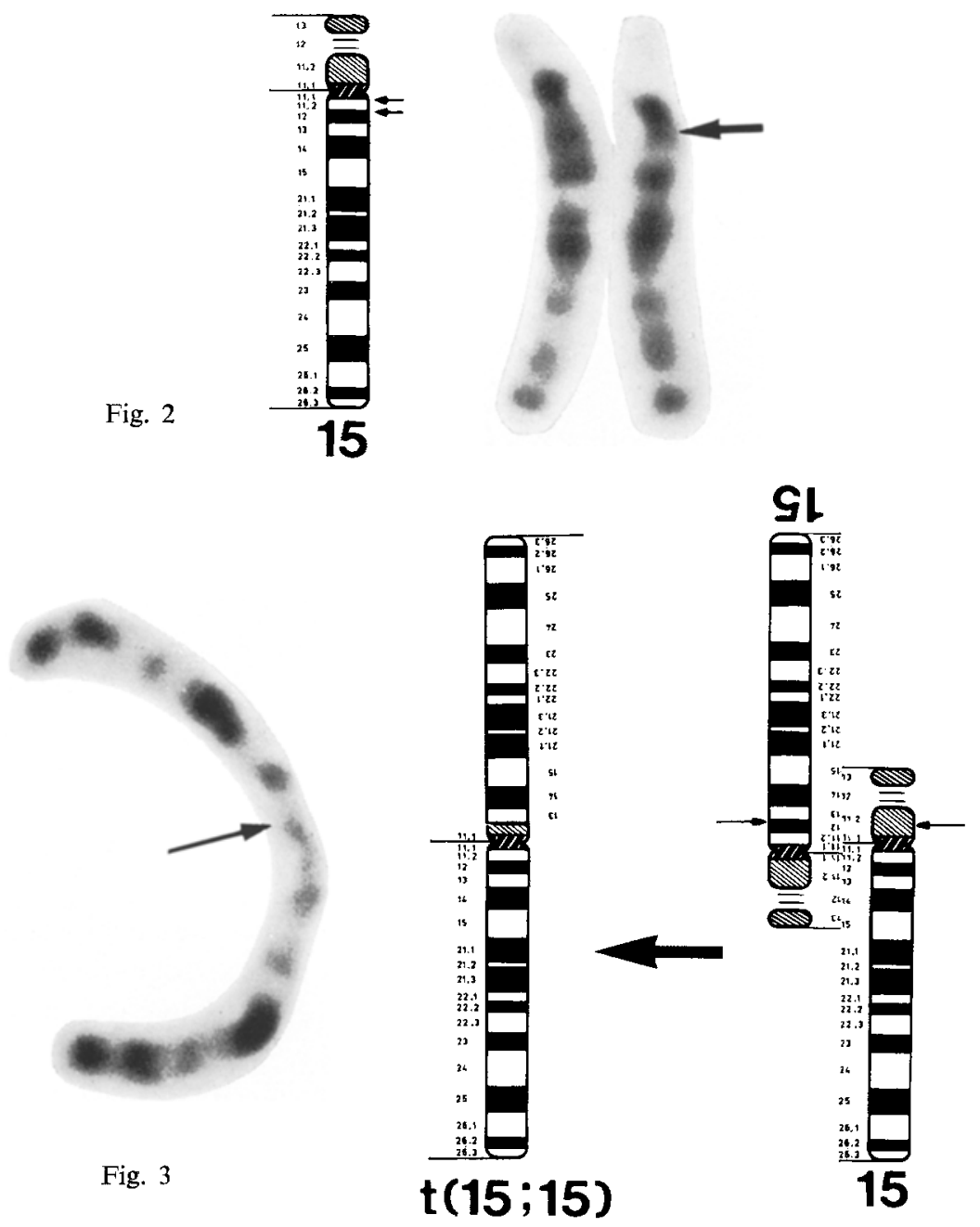

Vol. 29, No. 1, 1984 


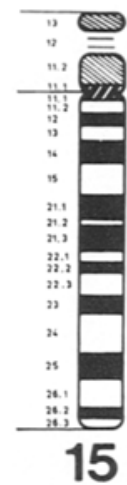

Fig. 4. Chromosome 15 from Case 10. The proximal region in both $15 \mathrm{q}$ is not deleted.

deletion in 15q11.2 and q12 bands (Fig. 3). One patient (Case 10) had a normal karyotype without any amount of deletion in both of $15 q$ (Fig. 4). All the parents examined had normal karyotypes.

\section{DISCUSSION}

Thirteen of the 14 patients with PWS $(93 \%$ ) had an interstitial deletion of proximal 15q. Although the segments involved in deletion were somewhat different in individual patients, the common affected region was confined to a sub-band $15 \mathrm{q} 11.2$ (ISCN, 1981).

Ledbetter and his colleagues (1982) examined 45 patients with PWS and found 23 cases with a deletion of a 15q11-12, one case with a balanced 15;15 translocation and one case with a mosaicism of $46 / 47,+\operatorname{idic}(15)(q 11)$. Thus, the patients studied by us had a $15 \mathrm{q}$ deletion more frequently than those of Ledbetter et al. This discrepancy may be due to the difference in the diagnostic criteria. In the present study, we selected only the patients who had typical clinical features of PWS including hypotonia, hypogonadism, obesity, mental retardation, a peculiar facies, short stature, and typical clinical courses.

In spite of our strict criteria in describing PWS, there was one patient who had a normal karyotype. This fact suggests three possibilities: submiroscopic deletions, mosaicism for an undetected population of cells with a deleted 15q, and etiological heterogeneity.

Four of the five infants with hypotonia had an interstitial deletion of $15 q$, and the remaining male infant had a 15;15 Robertsonian translocation with a deletion in 15q. The clinical diagnosis of PWS is usually made when a patient becomes obese with an uncontrollable hyperingestion in early childhood. As these five infants were 


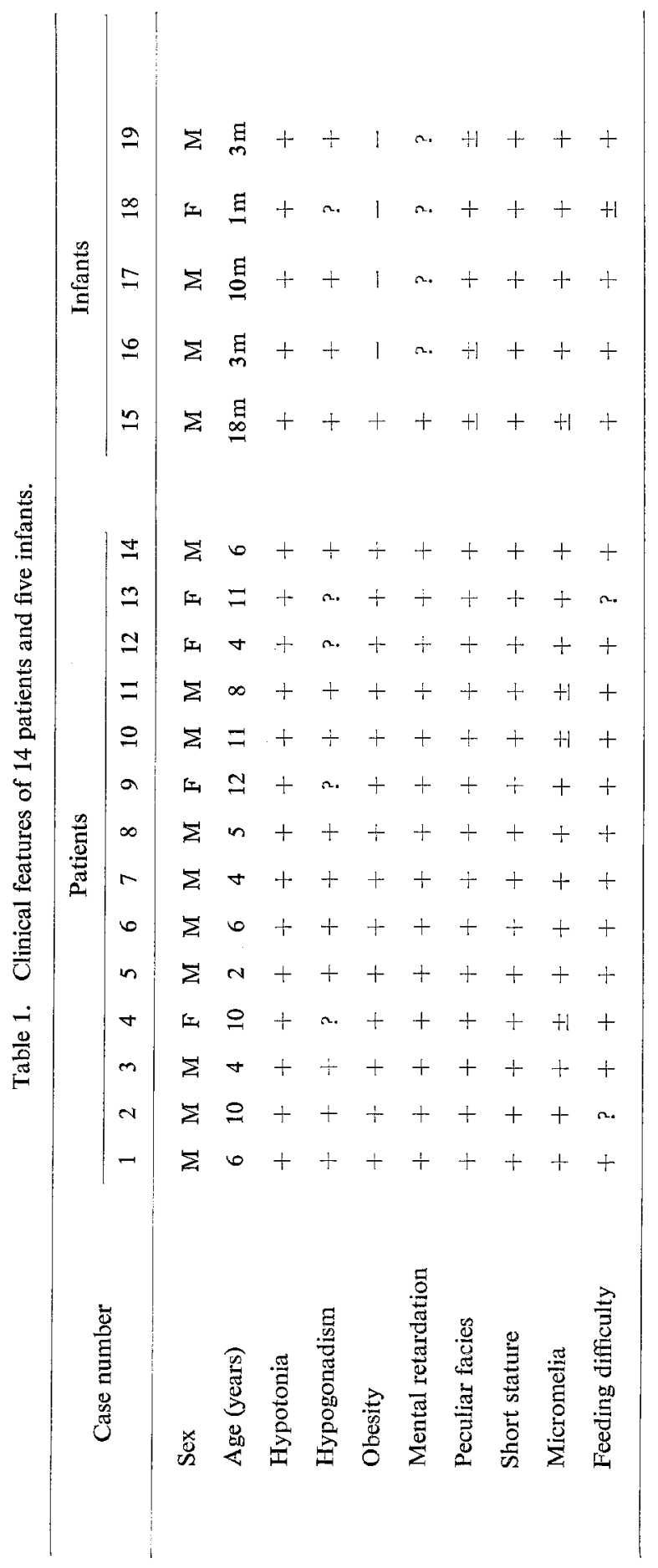

Vol. 29, No. 1, 1984 
not affected with obesity, the diagnosis of PWS had not been established until the recognition of the interstitial deletion of $15 \mathrm{q}$. The early diagnosis of PWS by a highresolution chromosome technique may have a merit in preventing patients from the custom of hyperphagia and subsequent severe obesity.

Acknowledgments. We thank Dr. S. Suwa (Director, Division of Endocrinclogy, Kanagawa Children's Medical Center) for referring patients to us, and Mr. D. P. Closser for reading the manuscript. This study was supported in part by Grant No. 82-0714 from National Center for Nervous, Mental and Muscular Disorders of the Ministry of Health and Welfare, Japan.

\section{REFERENCES}

Holm, V.A. 1981. The diagnosis of Prader-Willi syndrome. In Parader-Willi Syndrome, Holm, V.A., Sulzbacher, S.J., and Pipes, P.L., eds., University Park Press, Baltimore, pp. 27-36.

Ikeuchi, T. and Sasaki, M. 1979. Accumulation of early mitotic cells in ethidium bromide-treated human lymphocyte cultures. Proc. Jpn. Acad., 55: 15-18.

ISCN 1981: An International System for Human Cytogenetic Nomenclature-High-Resolution Banding. Cytogenet. Cell Genet. 31: 1-32.

Kouseff, B.G. 1982. The cytogenetic controversy in the Prader-Labhart-Willi syndrome. Am. J. Med. Genet. 13: 431-439.

Ledbetter, D.H., Riccardi, V.M., Airhart, S.D., Strobel, R.J., Keenan, B.S., and Crawford, J.D. 1981. Deletions of chromosome 15 as a cause of the Prader-Willi syndrome. New Engl. J. Med. 304: 325-329.

Ledbetter, D.H., Mascareilo, J.T., Riccardi, V.M., Harper, V.D., Airhart, S.D., and Strobel, R.J. 1982. Chromosome 15 abnormalities and the Prader-Willi syndrome: A follow-up report of 40 cases, Am. J. Hum. Genet. 34: 278-285.

McKusick, V.A. (ed.) 1978. Menderian Inheritance in Man, Johns Hopkins Univ. Press, Baltimore, London, p. 644 . 